\title{
Photonic RF and microwave channelizer based on a Kerr soliton crystal micro-comb
}

This paper was downloaded from TechRxiv (https://www.techrxiv.org).

\section{LICENSE}

CC BY 4.0

SUBMISSION DATE / POSTED DATE

30-10-2020 / 09-11-2020

\section{CITATION}

Moss, David (2020): Photonic RF and microwave channelizer based on a Kerr soliton crystal micro-comb. TechRxiv. Preprint. https://doi.org/10.36227/techrxiv.13167611.v1

DOI 


\title{
Photonic RF and microwave channelizer based on a Kerr soliton crystal micro-comb
}

\author{
Mengxi Tan ${ }^{1}$, Xingyuan $\mathrm{Xu}^{1,2}$, Jiayang $\mathrm{Wu}^{1}$, Andreas Boes ${ }^{3}$, Bill Corcoran ${ }^{2}$, Thach G. Nguyen ${ }^{3}$, Sai T. \\ $\mathrm{Chu}^{4}$, Brent E. Little ${ }^{5}$, Roberto Morandotti ${ }^{6}$, Senior Member IEEE, Arnan Mitchell ${ }^{3}$, Senior Member, IEEE, \\ and David J. Moss ${ }^{1}$, Fellow IEEE \\ ${ }^{I}$ Optical Sciences Centre, Swinburne University of Technology, Hawthorn, VIC 3122, Australia \\ ${ }^{2}$ Present Address: Dept. of Electrical and Computer Systems Engineering, Monash University, Clayton, 3800 VIC, Australia \\ ${ }^{3}$ School of Engineering, RMIT University, Melbourne, VIC 3001, Australia \\ ${ }^{4}$ Department of Physics and Material Science, City University of Hong Kong, Tat Chee Avenue, Hong Kong, China. \\ ${ }^{5} X i$ 'an Institute of Optics and Precision Mechanics Precision Mechanics of CAS, Xi'an, China. \\ ${ }^{6}$ INRS-Énergie, Matériaux et Télécommunications, 1650 Boulevard Lionel-Boulet, Varennes, Québec, J3X 1S2, Canada.
}

Index Terms-Microwave photonics, RF channelizer, micro-ring resonators, Kerr micro-combs.

\begin{abstract}
We report a 92 channel RF channelizer based on a 48.9 GHz integrated micro-comb that operates via soliton crystals, together with a passive high-Q ring resonator that acts as a periodic filter with an optical $3 \mathrm{~dB}$ bandwidth of $121.4 \mathrm{MHz}$. We obtain an instant RF bandwidth of $8.08 \mathrm{GHz}$ and $17.55 \mathrm{GHz}$ achieved through temperature tuning. These results represent a major advance to achieving fully integrated photonic RF spectrum channelizers with reduced low complexity, size, and high performance for digital-compatible signal detection and broadband analog signal processing.
\end{abstract}

\section{INTRODUCTION}

$\mathrm{M}$ odern RF systems require receivers as key components for electronic warfare, radar systems, satellite communications, and more [1-4], since they need to analyze and detect RF signals. However, the immediately accessible bandwidth (ie., without adjusting or thermal tuning for example) for systems to receive RF signals exceeds the capability of typical analog-to-digital converters (ADCs). Therefore, detecting and analyzing signals using flexible and powerful digital-domain tools requires that the broadband signal be sliced spectrally into segments that are compatible

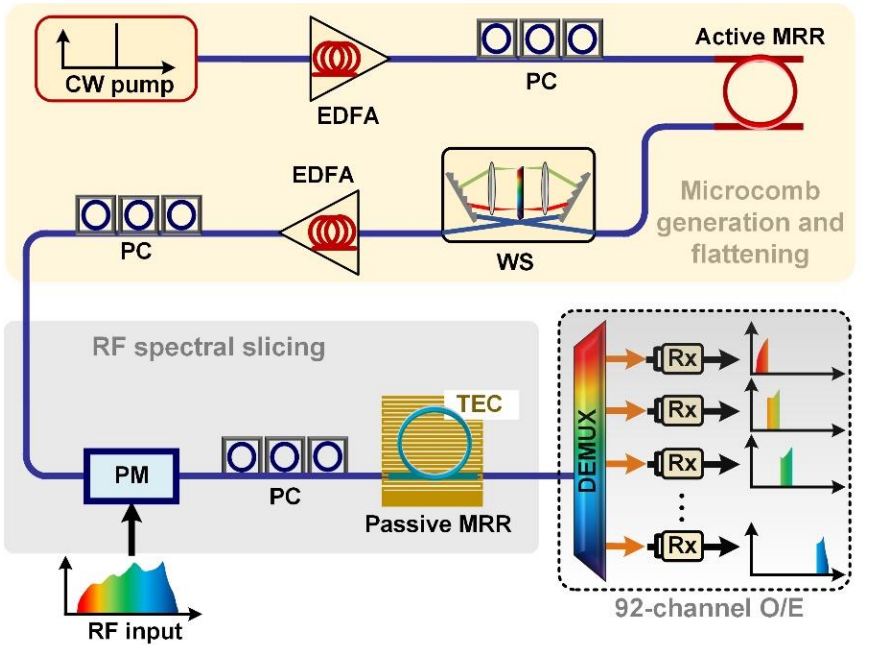

Fig. 1. Schematic of the broadband RF channelizer based on a soliton crystal microcomb. EDFA: erbium-doped fibre amplifier. PC: polarization controller. MRR: micro-ring resonator. WS: Waveshaper. PM: phase modulator. TEC: temperature controller. DEMUX: de-multiplexer. Rx: Receiver. with digital systems, [5] and this is the purpose of RF channelizers. While electronic based RF channelizers are limited by the electronic bottleneck in bandwidth, approaches based on photonics are attractive since they can provide very large bandwidths as well as low loss, together with very strong electromagnetic interference immunity.

A substantial body of work has been devoted towards developing RF channelizers based on photonics. These can generally be categorized into two different approaches. The first approach operates through separating the RF spectrum into channels with many high resolution and accurately spectrally positioned narrow passband optical filters [6]. While this is effective, it requires a larger footprint and cannot achieve extremely high RF resolution or bandwidth. The other approach to RF channelizers based on photonic uses a much more efficient and compact method that relies on a Vernier shift between a source of multiple wavelengths and an optical filter that is spectrally periodic, in order to realize an accurately progressively stepped broadband RF spectral channelization. A range of different approaches have been based on this strategy including stimulated Brillouin scattering [7], nonlinear optics in fibers [8], incoherent spectrally sliced sources [9], discrete arrays of laser diodes [10], electro-optic modulator generated frequency combs [5, 11], and others [12]. However, many of these suffer from drawbacks of one form or another including a limited number of channels, limited optical resolution, and difficulty in integrating the system due to the need discrete components.

Microcombs [13-17] have recently attracted significant attention, including CMOS compatible devices [18 - 23], since they can provide a large number of coherent channels in a very small (square millimeter) chip. They have formed the basis of a wide array of RF demonstrations [34-48, 35] including true RF time delays [36, 37], transversal filter based signal processing [38 - 43], RF frequency mixing and conversion [44], signal generators of various forms including phase-encoded generators [45], RF channelizers [47, 48] and many others [49-55]. We recently reported [47] a microcomb based RF channelizer that exhibited very high performance, where a $200 \mathrm{GHz}$ FSR microcomb provided twenty wavelengths across the $\mathrm{C}$ band. This microcomb was then filtered with a passive ring resonator with a FSR spacing of 48.9GHz. The RF signal was multicast onto the $200 \mathrm{GHz}$ 

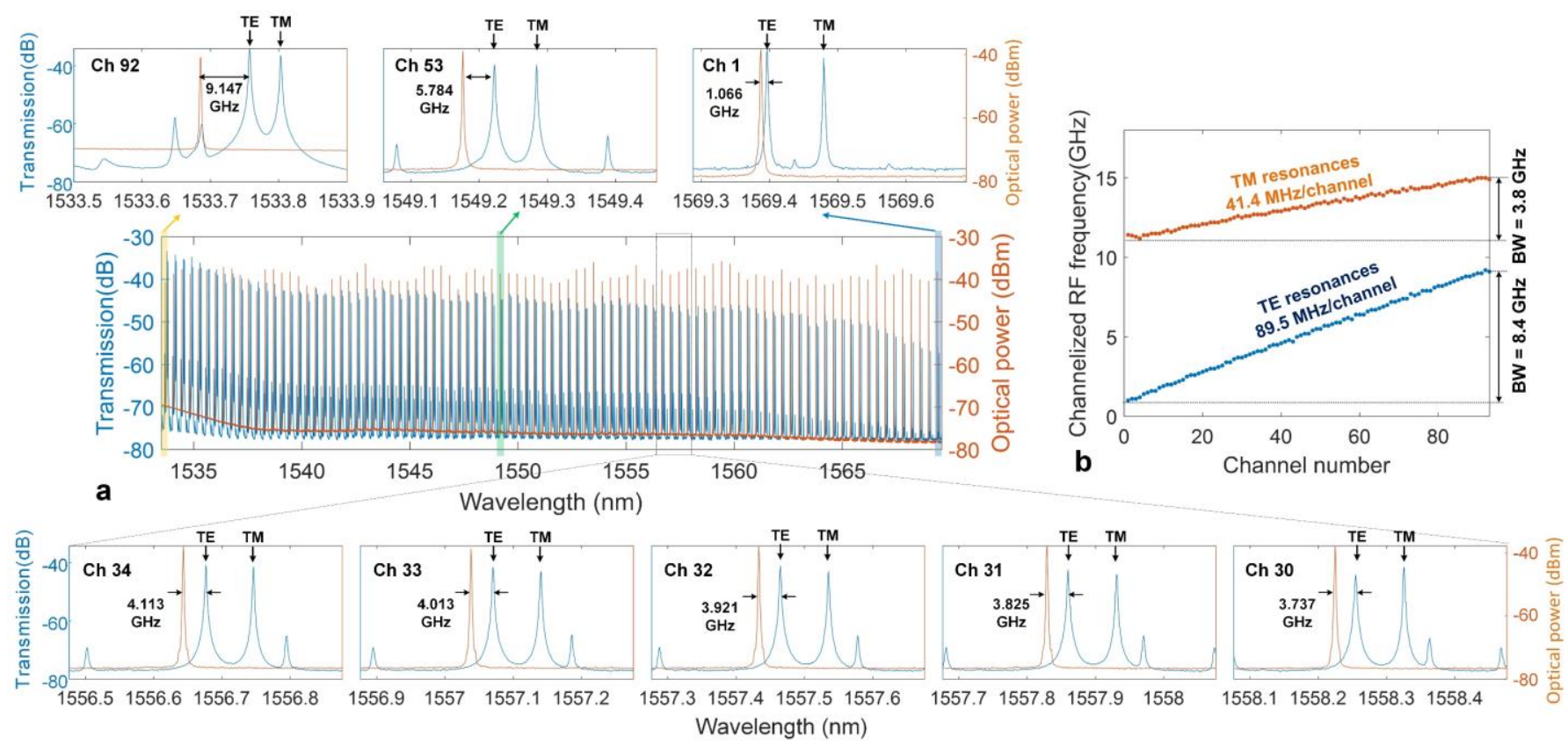

Fig. 2. (a) The measured optical spectrum of the micro-comb and drop-port transmission of passive MRR. (b) Extracted channelized RF frequencies of the 92 channels, calculated from the spacing between the comb lines and the passive resonances. Note that the labelled channelized RF frequencies in (a) is adopted from the accurate RF domain measurement using the Vector Network Analyzer.

microcomb which was subsequently sliced by every fourth passive ring resonator resonance. The net difference between the FSR of the microcomb and $4 \mathrm{x}$ the passive ring filter produced a frequency offset of $4.4 \mathrm{GHz}$. This resulted in the RF frequency step between the different RF channels, and since it was much larger than the $120 \mathrm{MHz}$ channel resolution, did not allow instantaneous spectral coverage of the full continuous RF spectrum. This resulted in a discontinuous RF operation band of 4 channels that could operate at one time, and so the overall bandwidth, given by the product of the slicing resolution and the channel number, was limited with "gaps". These gaps, however, could be filled in via temperature tuning the passive ring. The comparatively large $200 \mathrm{GHz}$ comb FSR yielded only 20 wavelengths across the C-band.

In this paper, [48] we demonstrate an RF channelizer based on photonics that achieves higher performance, accomplished through the use of two ring resonators with small, and closely matched, FSRs, at 49GHz. The first MRR generated a microcomb that operated via soliton crystals, while the second resonator acted as a passive filter, providing two major benefits. First, the very small FSR of $49 \mathrm{GHz}$ yielded 92 wavelengths across the $\mathrm{C}$ band which significantly increased the instant bandwidth to $8.08 \mathrm{GHz}$ - which is over $22 \mathrm{x}$ more than the previous device [47]. By using a ring with a closely matched FSRs to filter RF spectrum using Vernier shifting, a frequency step of the RF channelization lof 87.5 $\mathrm{MHz}$ was demonstrated. This led to a continuous channelization spectrum of the RF signal because the channelization step was less than the $121.4 \mathrm{MHz}$ optical resolution. The other major advantage of our approach is that we exploited parallel conversion of phase to intensitymodulation (PM - IM) for all wavelengths simultaneously which provided an instant RF output, while still in a stable and very small footprint without needing local oscillators. Lastly, the frequency range of operation of the channelizer could be tuned dynamically by varying the microcomb to passive ring offset frequency. We accomplished this by temperature tuning the passive ring, thus demonstrating RF channelization across a $17.55 \mathrm{GHz}$ total frequency range. This approach achieved high performance in a small footprint with low potential cost and complexity.

\section{OPERATION PRINCIPLE}

Figure 1 illustrates the operational principle of the RF channelizer. It comprises three sections - first the is produced and spectrally flattened with an optical waveshaper. With $N$ comb lines at a $\delta_{\text {OFC }}$ spacing, the $k^{\text {th }}(k=1,2,3, \ldots, 92)$ optical comb frequency is

$$
f_{\mathrm{OFC}}(k)=f_{\mathrm{OFC}}(1)+(k-1) \delta_{\mathrm{OFC}}
$$

with $f_{\mathrm{OFC}}(1)$ being the first comb line frequency on the long wavelength (red) side.

In the second module, the flattened comb lines were passed through an electro-optic phase modulator in order to multicast the RF signal onto all wavelengths. In the last module Finally, the replicated RF spectra are sliced by the passive MRR with an FSR of $\delta_{\mathrm{MRR}}$, with the resolution given by the $3 \mathrm{~dB}$ bandwidth of the resonator. As a result, the different RF channels have a center frequency given by

$$
\begin{aligned}
f_{\mathrm{RF}}(k) & =f_{\mathrm{MRR}}(k)-f_{\mathrm{OFC}}(k) \\
& =\left[f_{\mathrm{MRR}}(1)-f_{\mathrm{OFC}}(1)\right]+(k-1)\left(\delta_{\mathrm{MRR}}-\delta_{\mathrm{OFC}}\right)
\end{aligned}
$$

where $f_{\mathrm{RF}}(\mathrm{k})$ is the $k_{t h}$ channelized RF frequency, and $f_{\mathrm{MRR}}(\mathrm{k})$ is the passive ring's $k^{\text {th }}$ center frequency. Here, the relative difference between the $1^{\text {st }}$ comb line and adjacent passive ring resonance is given by $\left[f_{\mathrm{MRR}}(1)-f_{\mathrm{OFC}}(1)\right]$, which yields the offset RF channel frequency, and $\left(\delta_{\mathrm{OFC}}-\delta_{\mathrm{MRR}}\right)$ is the RF frequency step between adjacent wavelengths.

We used a combination of notch filtering with phase modulation to perforrm phase to intensity modulation conversion. Phase modulating first generated both lower and upper sidebands that had opposite phase. One of the 

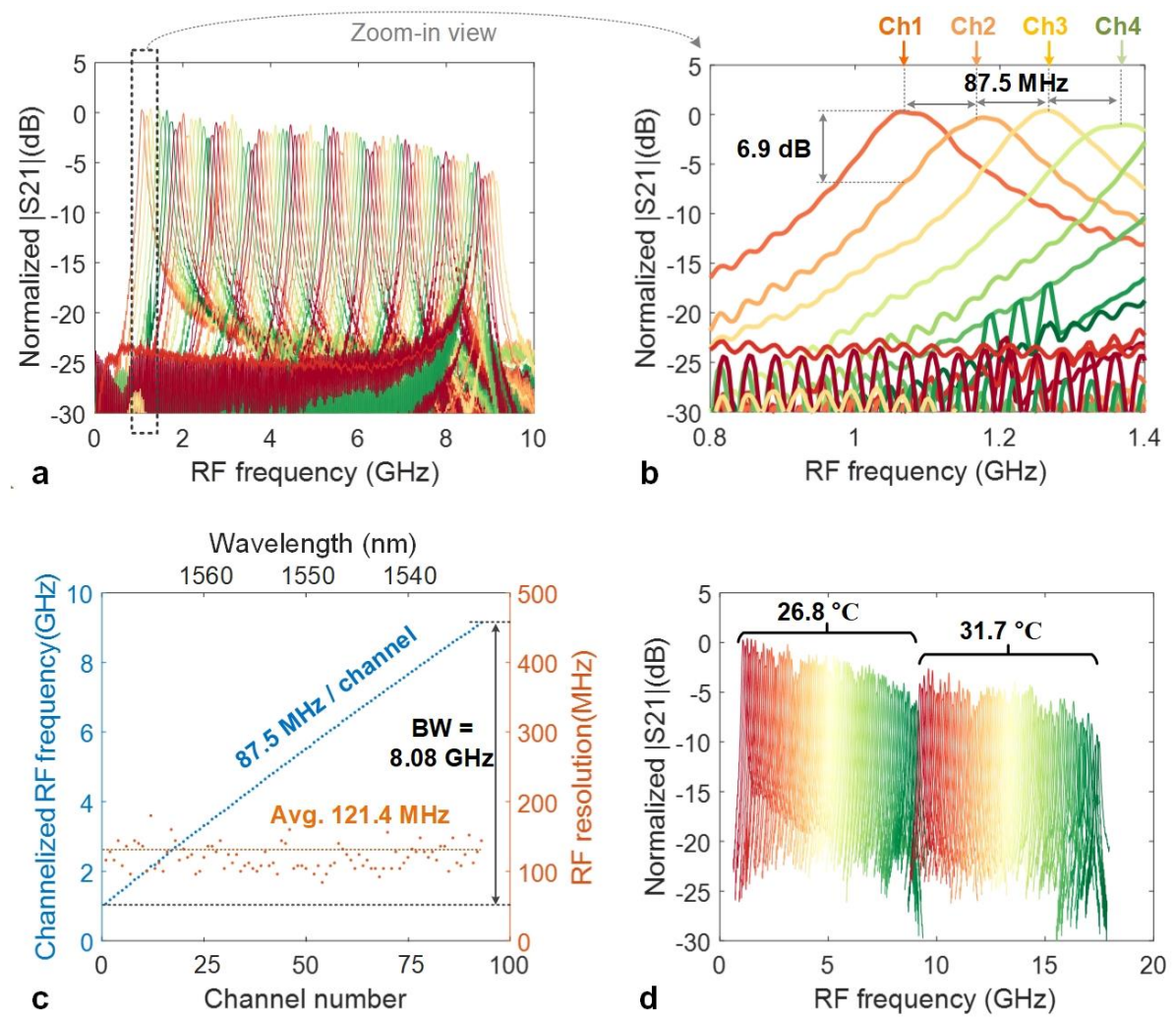

Fig. 3. RF transmission spectra of (a) the 92 channels and (b) a zoom-in of the first 4 channels. (b) Extracted channelized RF frequency and resolution. (d) Measured RF transmission spectra vs chip temperature of the passive MRR.

sidebands was then eliminated by the notch filter resonance, which left the other unsuppressed optical sideband to beat with the optical carrier after detection. Thus, this approach directly converted the modulation format from phase to single sideband intensity, providing $N$ parallel bandpass filters with a spectral resolution $\Delta f$ determined by the linewidth of the passive ring. The $\mathrm{RF}$ center frequencies $f_{\mathrm{RF}}(\mathrm{k})$ were given by the offset between the passive ring resonant frequencies and the comb lines $\left[f_{\mathrm{MRR}}(\mathrm{k})-f_{\mathrm{OFC}}(\mathrm{k})\right]$, given by Eq. $(2)$. As a result, the input RF spectrum was channelized into $N$ segments, each centered at $f_{\mathrm{RF}}(\mathrm{k})$ and with a bandwidth of $\Delta f$. This approach did not require local oscillators to perform coherent homodyne detection, and so it was much more stable and compact [48].

Finally, the wavelengths were separated and converted into the electric signals by separate photodetectors, thus yielding $N$ simultaneous RF channels with a spectral width of $\Delta f$, which is in the range of ADCs. After that, the channelized RF signals are converted into digital signals via an array of ADCs and processed with digital domain tools for further analysis.

\section{RESULTS}

The ring resonators were fabricated in Hydex which is CMOS compatible, and featured low propagation loss of 0.06 $\mathrm{dB}$ per $\mathrm{cm}$, a moderately high nonlinearity of $233 / \mathrm{W} / \mathrm{km}$ ) and no measurable nonlinear loss $[13,14]$. The rings had the same radius of $592 \mu \mathrm{m}$ with a $1.5 \times 2 \mu \mathrm{m}$ cross sections and Q's of greater than 1 million, with a total fibre to fibre insertion loss of $1.5 \mathrm{~dB}$. The active ring featured anomalous dispersion in the $\mathrm{C}$ band to support parametric gain, as well as having a mode crossing near $1556 \mathrm{~nm}$, needed to generate soliton crystal microcomb. The power of the pump was amplified to $2 \mathrm{~W}$ and then its wavelength was manually swept from blue to red to initiate the comb [56,57], that had a palm- like spectrum with a $48.9 \mathrm{GHz}$ spacing, producing 92 wavelengths across the $\mathrm{C}$-band. The microcomb was lownoise, coherent, and very easy to generate. All 92 comb lines were flattened and passed through the phase modulator, driven by the RF signal in order to multicast the RF signal onto the optical carriers at all wavelengths. The RF replicas were then sliced spectrally via the passive ring.

The passive ring transmission spectrum is shown in Fig. 2(a) measured with a broadband EDFA source. The RF channelized frequency $f_{\mathrm{RF}}(\mathrm{k})$, determined by the comb line to adjacent passive resonance spacing, decreased monotonically from blue to red. The total RF channelized frequency (Fig. 2(b)) had a $8.4 \mathrm{GHz}$ bandwidth for TE and $3.8 \mathrm{GHz}$ for TM, with steps of 89.5 (TE) and 41.4 (TM) MHz per channel. The channel resolution was $120 \mathrm{MHz}$ for TE polarization. The polarization was aligned to the TE mode of the passive ring and so the TM modes were not coupled into. The RF spectrum was then channelized into many segments over different wavelengths, which were separated into parallel spatial paths and detected individually. We used a Vector Network Analyzer to measure the RF transmission spectrum (Fig. 2 (a, b)). The channelizer SNR was $23.7 \mathrm{~dB}$, which can be improved by increasing the rejection of the passive optical ring filter. This can be achieved by optimizing the coupling coefficient for the bus waveguide to ring resonator.

The RF channel center frequencies (Fig. 3) had a $87.5 \mathrm{MHz}$ per channel step with an overall $8.08 \mathrm{GHz}$ instant bandwidth, agreeing with calculations based on the ring's spectrum. The average $\mathrm{RF}$ channel resolution obtained from the ring $3 \mathrm{~dB}$ bandwidth for the RF channel's transmission spectrum was 121.4 MHz. This high a resolution proves that this method is compatible with the modest bandwidths of most digital components. Finally, the operation band of the channelizer was variable from 1.006-9.147 GHz to $9.227-17.49 \mathrm{GHz}$, by 
temperature tuning the passive ring on millisecond timescales thus reaching an overall RF bandwidth of $16.48 \mathrm{GHz}$ (Fig. 3(d)). The maximum channelizer operation frequency (Nyquist zone) was $48.9 \mathrm{GHz} / 2=24.45 \mathrm{GHz}$. Larger spaced microcombs can increase the maximum RF to $100 \mathrm{GHz}$, but this comes with a tradeoff in the comb line number.

\section{CONCLUSION}

We report a RF channelizer with high performance based on Kerr microcombs that operate via soliton crystals. The 49GHz FSR of both the passive and active rings that closely matched each other produced 92 wavelengths over the Cband. This enabled a very large $8.08 \mathrm{GHz}$ instant RF bandwidth and maximum range of $17.49 \mathrm{GHz}$, achieved via temperature tuning. The channelizer had a high RF resolution of $121.4 \mathrm{MHz}$. This method is highly attractive to achieve instantaneous broadband RF signal detection and processing, towards photonic integrated RF system receivers.

\section{REFERENCES}

[1] J. Capmany and D. Novak, "Microwave photonics combines two worlds," Nat. Photonics, vol. 1, no. 6, pp. 319-330, Jun. 2007.

[2] J. P. Yao, "Microwave Photonics," J. Lightwave Technol., vol. 27, no. 1-4, pp. 314-335, Jan. 2009.

[3] S. L. Pan and J. P. Yao, "Photonics-Based Broadband Microwave Measurement," J. Lightwave Technol., vol. 35, no. 16, pp. 3498-3513, Aug. 2017.

[4] J. Azana, C. Madsen, K. Takiguchi, and G. Cincotti, "Guest editorial Optical signal processing," J. Lightwave Technol., vol. 24, no. 7, pp. 2484-2486, Jul. 2006.

[5] X. J. Xie, Y. T. Dai, Y. Ji, K. Xu, Y. Li, J. Wu, and J. T. Lin, "Broadband Photonic Radio-Frequency Channelization Based on a 39GHz Optical Frequency Comb," IEEE Photonic Tech. L., vol. 24, no. 8, pp. 661-663, Apr. 2012.

[6] S. T. Winnall, A. C. Lindsay, M. W. Austin, J. Canning, and A. Mitchell, "A microwave channelizer and spectroscope based on an integrated optical Bragg-grating Fabry-Perot and integrated hybrid fresnel lens system," IEEE T. Microw. Theory, vol. 54, no. 2, pp. 868872, Feb. 2006.

[7] W. Y. Xu, D. Zhu, and S. L. Pan, "Coherent photonic radio frequency channelization based on dual coherent optical frequency combs and stimulated Brillouin scattering," Opt. Eng., vol. 55, no. 4, Apr. 2016.

[8] S. Bres, S. Zlatanovic, A. O. J. Wiberg, J. Adleman, C. Huynh, E. Jacobs, J. Kvavle, and S. Radic, "Parametric Photonic Channelized RF Receiver," IEEE Photonic Tech. L., vol. 23, no. 6, pp. 344-346, Mar. 2011.

[9] X. H. Zou, W. Pan, B. Luo, and L. S. Yan, "Photonic approach for multiple-frequency-component measurement using spectrally sliced incoherent source," Opt. Lett., vol. 35, no. 3, pp. 438-440, Feb. 2010.

[10] F. A. Volkening, "Photonic channelized RF receiver employing dense wavelength division multiplexing," U.S. Patent 724583 3B1, 2007.

[11] X. J. Xie, Y. T. Dai, K. Xu, J. Niu, R. X. Wang, L. Yan, and J. T. Lin, "Broadband Photonic RF Channelization Based on Coherent Optical Frequency Combs and I/Q Demodulators," IEEE Photonics J., vol. 4, no. 4, pp. 1196-1202, Aug. 2012.

[12] Z. Li, X. M. Zhang, H. Chi, S. L. Zheng, X. F. Jin, and J. P. Yao, “A Reconfigurable Microwave Photonic Channelized Receiver Based on Dense Wavelength Division Multiplexing Using an Optical Comb," Opt. Commun., vol. 285, no. 9, pp. 2311-2315, May. 2012.

[13] L. Razzari, D. Duchesne, M. Ferrera, R. Morandotti, S. Chu, B. E. Little, and D. J. Moss, "CMOS-compatible integrated optical hyperparametric oscillator," Nat. Photonics, vol. 4, no. 1, pp. 41-45, Jan. 2010.

[14] D.J. Moss, R. Morandotti, A. L. Gaeta, and M. Lipson, "New CMOScompatible platforms based on silicon nitride and Hydex for nonlinear optics," Nat. Photonics, vol. 7, no. 8, pp. 597-607, Aug. 2013.

[15] A. L. Gaeta, M. Lipson, and T. J. Kippenberg, "Photonic-chip-based frequency combs," Nat. Photonics, vol. 13, no. 3, pp.158-169, Mar. 2019.

[16] T. J. Kippenberg, A. L. Gaeta, M. Lipson, and M. L. Gorodetsky, "Dissipative Kerr solitons in optical microresonators," Science, vol. 361, no. 6402, pp. 8083. 2018.
[17] A. Pasquazi et al., "Micro-Combs: A Novel Generation of Optical Sources", Physics Reports, vol. 729, pp. 1-81, Jan. 2018.

[18] M. Ferrera, L. Razzari, D. Duchesne, R. Morandotti, Z. Yang, M. Liscidini, J. E. Sipe, S. Chu, B. Little, and D. J. Moss, "Low-power CW nonlinear optics in doped silica glass integrated waveguide structures," Nat. Photonics, vol. 2, no. 12, pp. 737-740, Dec. 2008.

[19] M. Kues, C. Reimer, P. Roztocki, L. R. Cortes, S. Sciara, B. Wetzel, Y B. Zhang, A. Cino, S. T. Chu, B. E. Little, D. J. Moss, L. Caspani, J Azana, and R. Morandotti, "On-chip generation of high-dimensional entangled quantum states and their coherent control," Nature, vol. 546, no. 7660 , pp. 622-626, Jun. 2017.

[20] L. Caspani, C. Xiong, B. Eggleton, D. Bajoni, M. Liscidini, M. Galli, R. Morandotti, David J. Moss, "On-chip sources of quantum correlated and entangled photons", Nature: Light Science and Applications, vol. 6, e17100 (2017); doi: 10.1038/lsa.2017.100.

[21] M. Kues, C. Reimer, B. Wetzel, P. Roztocki, B. E. Little, S. T. Chu, T Hansson, E. A. Viktorov, D. J. Moss, and R. Morandotti, "Passively mode-locked laser with an ultra-narrow spectral width," Nat Photonics, vol. 11, no. 3, pp. 159-162. Mar. 2017.

[22] C. Reimer, M. Kues, P. Roztocki, B. Wetzel, F. Grazioso, B. E. Little, S. T. Chu, T. Johnston, Y. Bromberg, L. Caspani, D. J. Moss, and R. Morandotti, "Generation of multiphoton entangled quantum states by means of integrated frequency combs," Science, vol. 351, no. 6278, pp. 1176-1180, Mar. 2016

[23] C. Reimer et al "Cross-polarized photon-pair generation and bichromatically pumped optical parametric oscillation on a chip," Nat. Commun., vol. 6, Article: 8236 (2015).

[24] H. Bao et al., "Turing patterns in a fibre laser with a nested microresonator: robust and controllable micro-comb generation", Physical Review Research, vol. 2, 023395 (2020). DOI: 10.1103/PhysRevResearch.00.003000.

[25] H. Bao, et al., "Laser Cavity-Soliton Micro-Combs", Nature Photonics, vol. 13, no. 6, pp. 384-389 (2019). DOI:10.1038/s41566-019-0379-5.

[26] S. Sciara et al., "Optimal entanglement witnesses for N-partite d-level quantum states customized to restrictive measurement settings", Physical Review Letters, vol. 122, 120501 (2019). DOI: 10.1103/PhysRevLett.122.120501.

[27] M. Kues, et. al., "Passively modelocked laser with an ultra-narrow spectral width", Nature Photonics, vol. 11, no. 3, 159 (2017). DOI:10.1038/nphoton.2016.271685.

[28] M. Kues, C. Reimer, A. Weiner, J. Lukens, W. Munro, D. J. Moss, and R. Morandotti, "Quantum Optical Micro-combs", Nature Photonics, vol. 13, no. 3, pp. 170-179 (2019). DOI:10.1038/s41566-019-0363-0.

[29] P. Roztocki et al., "Complex quantum state generation and coherent control based on integrated frequency combs", Journal of Lightwave Technology, vol. 37, no. 2, pp. 338-347 (2019).

[30] C. Reimer et al., "High-dimensional one-way quantum processing implemented on d-level cluster states", Nature Physics, vol. 15, no. 2, pp. 148-153 (2019). DOI:10.1038/s41567-018-0347-x

[31] B. Wetzel et al., "Smart supercontinuum sources: Customizing nonlinear optical interactions via adaptive temporal pulse-splitting", Nature Communications, vol. 9, 4884, Nov. 20 (2018).

[32] H. Bao et al., "Type-II Micro-Comb Generation in a Filter-Driven Four Wave Mixing Laser", Photonics Research Journal, vol. 6, no. 5, pp. B67-B73 (2018)

[33] A. Pasquazi, et al., "Sub-picosecond phase-sensitive optical pulse characterization on a chip", Nature Photonics, vol. 5, no. 10, pp. 618 623 (2011). DOI: 10.1038/nphoton.2011.199.

[34] J. Wu, X. Xu, T. G. Nguyen, S. T. Chu, B. E. Little, R. Morandotti, A Mitchell, and D. J. Moss, "RF Photonics: An Optical Microcombs' Perspective," IEEE Journal of Selected Topics in Quantum Electronics, vol. 24, no. 4, pp. 1-20. 2018.

[35] X. Xu, M. Tan, J. Wu, R. Morandotti, A. Mitchell, and D. J. Moss, "Microcomb-based photonic RF signal processing," IEEE Photonics Technology Letters, vol. 31, no. 23, pp. 1854-1857. 2019.

[36] X. Xu, J. Wu, T. G. Nguyen, T. Moein, S. T. Chu, B. E. Little, R. Morandotti, A. Mitchell, and D. J. Moss, "Photonic microwave true time delays for phased array antennas using a 49 GHz FSR integrated optical micro-comb source," Photonics Research, vol. 6, no. 5, pp. B30-B36, May 1. 2018.

[37] X. Xue, Y. Xuan, C. Bao, S. Li, X. Zheng, B. Zhou, M. Qi, and A. M. Weiner, "Microcomb-Based True-Time-Delay Network for Microwave Beamforming With Arbitrary Beam Pattern Control," Journal of Lightwave Technology, vol. 36, no. 12, pp. 2312-2321, Jun. 2018.

[38] X. Xu, J. Wu, T. G. Nguyen, M. Shoeiby, S. T. Chu, B. E. Little, R Morandotti, A. Mitchell, and D. J. Moss, "Advanced RF and microwave functions based on an integrated optical frequency comb source," Optics Express, vol. 26, no. 3, pp. 2569-2583, Feb 5. 2018. 
[39] X. Xu, M. Tan, J. Wu, T. G. Nguyen, S. T. Chu, B. E. Little, R. Morandotti, A. Mitchell, and D. J. Moss, "Advanced Adaptive Photonic RF Filters with 80 Taps Based on an Integrated Optical Micro-Comb Source," Journal of Lightwave Technology, vol. 37, no. 4, pp. 1288-1295, Feb. 2019.

[40] X. X. Xue, Y. Xuan, H. J. Kim, J. Wang, D. E. Leaird, M. H. Qi, and A. M. Weiner, "Programmable Single-Bandpass Photonic RF Filter Based on Kerr Comb from a Microring," Journal of Lightwave Technology, vol. 32, no. 20, pp. 3557-3565, Oct 15. 2014.

[41] X. Xu, J. Wu, M. Shoeiby, T. G. Nguyen, S. T. Chu, B. E. Little, R. Morandotti, A. Mitchell, and D. J. Moss, "Reconfigurable broadband microwave photonic intensity differentiator based on an integrated optical frequency comb source," APL Photonics, vol. 2, no. 9, 096104 Sep. 2017.

[42] M. Tan, X. Xu, B. Corcoran, J. Wu, A. Boes, T.G. Nguyen, S.Chu, B. Little, R. Morandotti, A. Mitchell, and D.J. Moss, "Microwave and RF photonic fractional Hilbert transformer based on a $50 \mathrm{GHz}$ Kerr microcomb," J. Lightwave Technology, vol. 37, no. 24, pp. 6097-6104. 2019

[43] M. Tan, X. Xu, B. Corcoran, J. Wu, A. Boes, T. G. Nguyen, S. T. Chu, B. E. Little, R. Morandotti, A. Mitchell, and D. J. Moss, "RF and microwave fractional differentiator based on photonics," IEEE Transactions on Circuits and Systems II: Express Briefs, Vol. 67, No.11, pp. 2767-2771. 2020. DOI:10.1109/TCSII.2020.2965158

[44] X. Xu, J. Wu, M. Tan, T. G. Nguyen, S. Chu, B. Little, R. Morandotti, A. Mitchell, and D. J. Moss, "Micro-comb based photonic local oscillator for broadband microwave frequency conversion," Journal of Lightwave Technology, vol. 38, no. 2, pp. 332-338. 2020.

[45] X. Xu, M. Tan, J. Wu, A. Boes, B. Corcoran, T. G. Nguyen, S. T. Chu, B. Little, R. Morandotti, A. Mitchell and D. J. Moss, "Photonic RF phase-encoded signal generation with a microcomb source," Journal of Lightwave Technology, vol. 38, no. 7, pp. 1722-1727. 2020.

[46] X. Xu, M. Tan, J. Wu, T. G. Nguyen, S. T. Chu, B. E. Little, R. Morandotti, A. Mitchell, and D. J. Moss, "High performance RF filters via bandwidth scaling with Kerr micro-combs," APL Photonics, vol. 4, no. 2, pp. 026102. 2019.

[47] X. Xu, J. Wu, T. G. Nguyen, S. Chu, B. Little, A. Mitchell, R. Morandotti, and D. J. Moss, "Broadband RF Channelizer based on an Integrated Optical Frequency Kerr Comb Source," Journal of Lightwave Technology, vol. 36, no. 19, pp. 4519 - 4526. 2018

[48] X. Xu et al, "Broadband photonic radio frequency channelizer with 90 channels based on a soliton crystal microcomb", Journal of Lightwave Technology, vol. 38., no. 18, 5116 - 5121, Sept. 15, 2020.

[49] X. Xu et al, "Photonic perceptron based on a Kerr microcomb for scalable high speed optical neural networks", Laser and Photonics $\begin{array}{llllll}\text { Reviews, } & \text { vol. } \quad 14, \quad \text { no. } & 8, & 2000070 \quad \text { (2020). }\end{array}$ DOI:10.1002/lpor.202000070.

[50] M. Tan et al, "Photonic RF and microwave filters based on 49GHz and 200GHz Kerr microcombs", Optics Communications, vol. 465, Article: 125563. 2020. DOI:10.1016/j.optcom.2020.125563.

[51] X. Xu et al, "Photonic RF and microwave integrator with soliton crystal microcombs", IEEE Transactions on Circuits and Systems: Express Briefs, Early Access (2020). DOI:10.1109/TCSII.2020.2995682

[52] M. Tan et al, "Photonic RF arbitrary waveform generator based on a soliton crystal micro-comb source", Journal of Lightwave Technology, vol. 38, no. 22, pp. 6221-6226, Oct 22 (2020). DOI:10.1109/JLT.2020.3009655.

[53] B. Corcoran et al, "Ultra-dense optical data transmission over standard fiber with a single chip source", Nature Communications, vol. 11, Article: 2568, May 22 (2020). DOI:10.1038/s41467-020-16265-x.

[54] T. G. Nguyen et al, "Integrated frequency comb source based Hilbert transformer for wideband microwave photonic phase analysis", Optics Express, vol. 23, no. 17, 22087 (2015). DOI:10.1364/OE.23.022087.

[55] M. Tan et al, "RF and microwave high bandwidth signal processing based on Kerr Micro-combs", Advances in Physics X, Vol 5, (2020). DOI:10.1080/23746149.2020.1838946.

[56] D. C. Cole, E. S. Lamb, P. Del'Haye, S. A. Diddams, and S. B. Papp, "Soliton crystals in Kerr resonators," Nature Photonics, vol. 11, no. 10, pp. 671. 2017.

[57] W. Wang, Z. Lu, W. Zhang, S. T. Chu, B.Little, L. Wang, X. Xie, M. Liu, Q. Yang, and L. Wang, "Robust soliton crystals in a thermally controlled microresonator," Optics letters, vol. 43, no. 9, pp. 20022005. 2018. 